\title{
Évolution nycthémérale des produits de la fermentation bactérienne dans le tube digestif du lapin en croissance. Relations avec la teneur en lignines de la ration
}

\author{
T. GIDENNE \\ Laboratoire de Recherches sur l'Elevage du Lapin \\ Chemin de Borde-Rouge, Auzeville, B.P. 27, F 31326 Castanet-Tolosan Cedex
}

\section{Résumé}

Trois lots de 36 lapereaux ont reçu à volonté des rations à teneur élevée en lignines Van-Soest ( 7 à 17 p. 100 , tabl. 1). Après abattage d'animaux répartis toutes les 3 heures durant 24 heures, les AGV, l'azote ammoniacal et le lactate ont été dosés à 4 niveaux du tube digestif : Estomac, Iléon (terminal), Cæcum, Rectum. Les rations expérimentées ne mettent pas en évidence un effet des lignines sur les teneurs (tabl. 2) ou les proportions (tabl. 3), des produits de fermentations cacocoliques. La teneur en ammoniac est élevée dans l'iléon $(38,8 \mathrm{mM} / \mathrm{l})$ et le cæcum $(24,8 \mathrm{mM} / \mathrm{l})$. Elle augmente dans le rectum en période de cæcotrophie (tabl. 7), mais ne varie pas au cours du nycthémère dans les autres sites de prélèvement. Les concentrations en AGV sont maximum dans le rectum et le cæecum $(50$ à $100 \mathrm{mM} / 1)$. L'acétate est l'AGV majeur (70 à 90 p. 100) suivi du butyrate (5- 16 p. 100) et du propionate $(0$ à 6 p. 100). En période de cacotrophie, la teneur en AGV totaux (fig. 1) baisse dans le cæcum et s'élève dans l'estomac et le rectum. En même temps, la qualité des fermentations est modifiée : hausse de la proportion de butyrate et baisse de la proportion d'acétate (fig. 3, estomac, rectum).

Mots clés : Acides gras volatils, ammoniac, lapin, cacotrophie, lignines.

\section{Introduction}

Dans le cadre d'une étude plus générale concernant l'utilisation digestive des parois végétales par le lapin, un travail précédent nous a permis de souligner l'incidence de la cæcotrophie sur l'évolution nycthémérale quantitative et qualitative ( $\mathrm{pH}$, cæcotrophes) des contenus digestifs (GIDENNE \& Lebas, 1984).

Plusieurs travaux ont déjà montré chez le lapin l'importance des fermentations microbiennes dans l'ensemble cæcum-côlon (Cools \& JeniauX, 1961; Hoover \& HeitmanN, 1972...), et les variations de production d'acides gras volatils (AGV) pendant la journée (Le BARs et al., 1971 ; VernaY, 1985). Mais en fait, nous n'avons que peu d'informations sur les variations de ces produits de fermentations au cours d'un nycthémère complet. 
La composition de la ration, en particulier la teneur en constituants pariétaux, est un facteur majeur, intervenant tant sur le développement que sur la qualité des fermentations digestives. Pourtant, l'influence de la composition des constituants de la paroi (hémicelluloses, cellulose, lignines) n'a été que rarement étudiée chez le lapin. Ainsi, les lignines (considérées comme indigestibles) constituent-elles une barrière physique à l'attaque microbienne de la paroi végétale (AKIN et al., 1974 ; JUNG \& FAHEY, 1983 a), en raison des liaisons qu'elles forment avec les autres constituants pariétaux. Elles ont également un effet inhibiteur sur les fermentations, en fonction de leur composition en monomères phénoliques (AKIN, 1982 ; JUNG \& FAHEY, 1983 b).

Notre étude se propose donc de tester la présence de ces effets sur les fermentations cæco-coliques du lapin en croissance, recevant une ration à teneur élevée en lignines provenant de pellicules de colza et de luzerne déshydratée. Elle nous permet également de préciser l'évolution nycthémérale des produits de la fermentation à différents niveaux du tube digestif.

\section{Matériel et méthodes}

\section{A. Matériel animal et alimentation}

Au total, 3 lots de 36 lapereaux des deux sexes, de race Néozélandaise Blanche ont été élevés en cage individuelle dans un local éclairé $12 / 24$ h (7 h 30 à 19 h 30). Chaque lot a reçu à volonté pendant 20 jours, un aliment granulé riche en constituants pariétaux (20 p. 100 de cellulose Van-Soest), dont le principal facteur de variation entre lots est le taux de lignine Van-Soest (7,4 à 16,8 p. 100). La composition chimique et centésimale des 3 régimes est portée au tableau 1.

\section{B. Prélèvements des contenus digestifs}

Les animaux, à l'âge de 9 semaines, ont été sacrifiés par dislocation cervicale, par séries successives situées toutes les 3 heures, de $9 \mathrm{~h}$ le matin à $9 \mathrm{~h}$ le lendemain matin, soit 4 lapins par lot et par point horaire. Immédiatement après sacrifice, l'estomac, les 60 derniers centimètres de l'iléon, le cæcum et les 30 derniers centimètres du rectum ont été pesés pleins, puis la totalité des contenus a été prélevée. Le pH a été déterminé in situ immédiatement après la pesée de l'organe plein, en 2 sites pour l'estomac (fundus et antrum), en un seul pour le cæecum. Les résultats concernant l'évolution nycthémérale des contenus digestifs (poids frais, poids sec, $\mathrm{pH} . .$. ) et la pratique de la cæcotrophie chez ces lapins ont déjà été publiés (GIDENNE \& LEBAS, 1984).

\section{Dosages}

Un échantillon moyen, par lot, de digesta frais a été constitué à chaque heure pour chaque site de prélèvement, puis il a été acidifié $\left(\mathrm{H}_{3} \mathrm{PO}_{4} 5\right.$ p. 100 et $\mathrm{HgCl}_{2}$ 1 p. 100) et conservé à $-20^{\circ} \mathrm{C}$. Après décongélation, il a été centrifugé et le surnageant repris en vue du dosage des AGV par chromatographie en phase gazeuse (CPG) (Jouany, 1982). Cette technique permet également de détecter la présence de lactate $(<1 \mathrm{mM} / \mathrm{l})$ dans les digesta; par contre, les valeurs des concentrations sont 


\section{Tableau 1}

Composition des régimes expérimentaux.

Composition of experimental diets.

\begin{tabular}{|c|c|c|c|}
\hline \multirow{2}{*}{$\begin{array}{c}\text { Analyse chimique (\% M.S.) } \\
\text { Chemical analysis }\end{array}$} & \multicolumn{3}{|c|}{ Aliments - Diets } \\
\hline & B & $\mathbf{M}$ & $\mathbf{H}$ \\
\hline $\begin{array}{l}\text { MAT }(\mathrm{N} \times 6,25) \ldots \ldots \ldots \cdots \cdots \cdots \\
\text { Crude protein }(N \times 6,25)\end{array}$ & 16,75 & 16,81 & 17,06 \\
\hline $\begin{array}{l}\text { Amidon } \\
\text { Starch }\end{array}$ & 2,42 & 2,10 & 2,46 \\
\hline $\begin{array}{l}\text { Energie brute }(\mathrm{Kcal} / \mathrm{kg} \text { M.S. }) \\
\text { Gross energy }(\text { kcal } / \mathrm{kg} \text { D.M. })\end{array}$ & 4564 & 4633 & 4645 \\
\hline $\begin{array}{l}\text { Matières minérales } \ldots \ldots \ldots \ldots \ldots \ldots \\
\text { Minerals }\end{array}$ & 8,80 & 9,43 & 10,75 \\
\hline $\begin{array}{l}\text { Hemicellulose } \\
\text { Hemicellulose }\end{array}$ & 14,70 & 13,40 & 12,40 \\
\hline $\begin{array}{l}\text { Cellulose } \\
\text { Cellulose }\end{array}$ & 24,80 & 23,50 & 23,60 \\
\hline $\begin{array}{l}\text { Lignine } \\
\text { Lignin }\end{array}$ & 7,40 & 12,80 & 16,00 \\
\hline Composants - Components & & & \\
\hline $\begin{array}{l}\text { Luzerne déshydratée } \ldots \ldots \ldots \ldots \ldots \ldots \\
\text { Dehydrated lucerne }\end{array}$ & 89,78 & 73,00 & 55,41 \\
\hline $\begin{array}{l}\text { Pellicules colza } \ldots \ldots \ldots \ldots \ldots \ldots \ldots \\
\text { Rapeseed hulls }\end{array}$ & - & 19,00 & 39,00 \\
\hline $\begin{array}{l}\text { Huile de colza } \ldots \ldots \ldots \ldots \ldots \ldots \ldots \ldots \\
\text { Rapeseed oil }\end{array}$ & 4,38 & 2,29 & - \\
\hline $\begin{array}{l}\text { Tourteau de soja } \ldots \ldots \ldots \ldots \\
\text { Soyabean meal }\end{array}$ & 4,00 & 4,00 & 4,00 \\
\hline $\begin{array}{l}\text { Minéraux et vitamines } \ldots \ldots \ldots \\
\text { Minerals and vitamins }\end{array}$ & 1,30 & 1,30 & 1,30 \\
\hline $\begin{array}{l}\text { Phosphate bicalcique } \ldots \ldots \\
\text { Calcium diphosphate }\end{array}$ & 0,40 & 0,35 & 0,29 \\
\hline $\begin{array}{l}\mathrm{dl} \text { méthionine } \\
d l \text { methionine }\end{array}$ & 0,14 & 0,06 & 一 \\
\hline
\end{tabular}




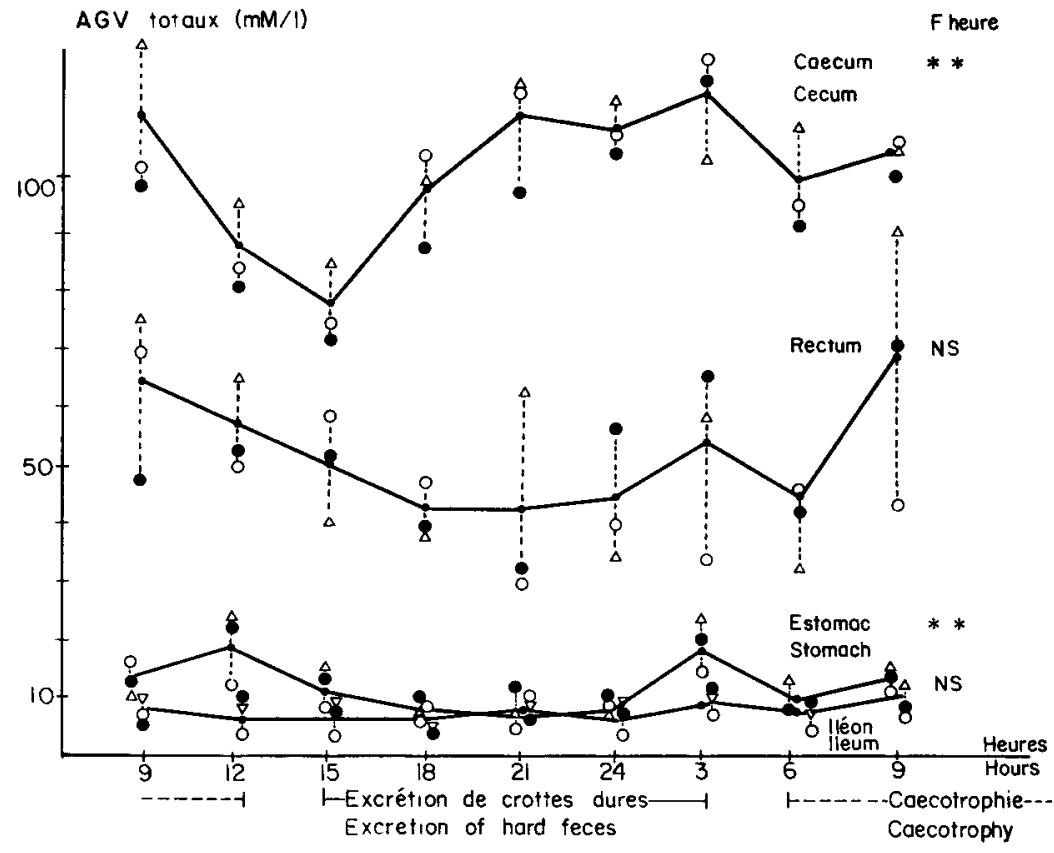

$\begin{array}{lll}\text { Régime 1: } & \text { Régime 2: } \\ \text { Diet | } & \text { Dieł } 2 & \text { Régime } 3: \bullet \\ \text { Diet } 3\end{array}$

FIG. 1

Evolution nycthémérale de la teneur en AGV totaux en fonction du lieu de prélèvement.

Circadian variation of total VFA concentration at the different collection sites.

Tableau 2

Teneurs moyennes ( $\mathrm{mM} / \mathrm{l})$ en A.G.V. totaux et en azote ammoniacal, aux différents sites de prélèvement, en fonction du régime alimentaire.

Mean concentrations $(\mathrm{mM} / \mathrm{l})$ of total V.F.A. and ammonia nitrogen at different collection sites according to the diet.

\begin{tabular}{|c|c|c|c|c|c|c|}
\hline \multirow{2}{*}{$\begin{array}{l}\text { Composants } \\
\text { Components }\end{array}$} & \multirow{2}{*}{$\begin{array}{c}\text { Site } \\
(2)\end{array}$} & \multicolumn{3}{|c|}{ Aliments - Diets } & \multirow{2}{*}{$\mathrm{CV} \%$} & \multirow{2}{*}{$\begin{array}{l}\text { Effet } \\
\text { Alim. } \\
(\mathrm{F}) \\
(3)\end{array}$} \\
\hline & & 1 & 2 & 3 & & \\
\hline $\begin{array}{l}\text { A.G.V. totaux } \\
\text { Total V.F.A. }\end{array}$ & $\begin{array}{l}\text { Estomac } \ldots \\
\text { Iléon } \ldots . . \\
\text { Cæcum } \ldots \\
\text { Rectum }\end{array}$ & $\begin{array}{r}13,0 \\
7,7 \\
97,4 \\
54,5\end{array}$ & $\begin{array}{r}9,6 \\
6,0 \\
103,8 \\
48,2\end{array}$ & $\begin{array}{r}13,5 \\
8,7 \\
108,9 \\
57,4 \\
\end{array}$ & $\begin{array}{r}23,7 \\
24,5 \\
7,3 \\
27,2\end{array}$ & $\begin{array}{c}* \\
* \\
* \\
\text { N.S. }\end{array}$ \\
\hline $\begin{array}{l}\text { Azote } \\
\text { (ammoniacal) } \\
\text { (Ammonia } \\
\text { nitrogen) }\end{array}$ & $\begin{array}{l}\text { Estomac } \ldots \\
\text { Iléon } \ldots . \\
\text { Cæcum } \ldots \\
\text { Rectum } \ldots\end{array}$ & $\begin{array}{l}11,3 \\
37,6 \\
23,6 \\
22,5\end{array}$ & $\begin{array}{l}10,8 \\
36,9 \\
23,0 \\
18,4\end{array}$ & $\begin{array}{l}12,9 \\
41,9 \\
27,6 \\
25,9\end{array}$ & $\begin{array}{r}21,7 \\
7,3 \\
24,0 \\
27,7\end{array}$ & $\begin{array}{l}\text { N.S. } \\
* * * \\
\text { N.S. } \\
\text { N.S. }\end{array}$ \\
\hline
\end{tabular}

(1) Rapport entre l'écart type résiduel intra lot et la moyenne générale.

Ratio between within-group residual standard error and general mean.

(2) Estomac - Stomach; Iléon - Ileum;Caecum - Caecum; Rectum - Rectum.

(3) Effect of the diet. 
peu précises : écart-type de la mesure $=2,5 \mathrm{mM} / \mathrm{l}$ (Jouany, communication personnelle). Nous avons constitué un second échantillon moyen dilué dans $\mathrm{NaCl} 12,5$ p. 100 puis congelé, sur lequel l'azote ammoniacal a été dosé en continu (après dialyse) selon la méthode de Weatherburn (1967). Les glucides pariétaux ont été dosés selon la méthode de Van Soest et Wine (1967). Le terme Lignines employé dans le texte s'applique donc au résidu obtenu selon ce procédé.

\section{Résultats et discussion}

\section{A. Effets moyens des aliments}

La teneur en lignines de nos rations influence peu la teneur moyenne en AGV totaux (tabl. 2), que ce soit aux niveaux stomacal, iléal ou rectal. Mais au niveau cæcal, nous pouvons distinguer une baisse légère de la teneur en AGV (-10 p. 100) lorsque le taux de lignines de la ration diminue. L'effet «lignines » n'est pas non plus visible sur les proportions molaires des principaux AGV, quel que soit le lieu de prélèvement dans l'intestin (tabl. 3). Par contre, dans l'estomac, nous observons une

\section{TABLEAU 3}

Proportions molaires moyennes des A.G.V. aux différents sites de prélèvements, en fonction du régime alimentaire.

Mean molar proportions of V.F.A., at different collection sites according to the diet.

\begin{tabular}{|c|c|c|c|c|c|c|}
\hline \multirow{2}{*}{$\underset{\%}{\text { A.G.V. }}$} & \multirow{2}{*}{$\begin{array}{c}\text { Site } \\
(3)\end{array}$} & \multicolumn{3}{|c|}{ Aliments - Diets } & \multirow{2}{*}{$\underset{111}{\mathrm{CV}} \%$} & \multirow{2}{*}{$\begin{array}{l}\text { Effet } \\
\text { Alim. } \\
(\mathrm{F}) \\
(2)\end{array}$} \\
\hline & & 1 & 2 & 3 & & \\
\hline $\begin{array}{l}\text { Acétate } \\
\text { Acetate }\end{array}$ & $\begin{array}{l}\text { Estomac } \ldots \\
\text { Iléon } \ldots \\
\text { Cxcum } \\
\text { Rectum }\end{array}$ & $\begin{array}{l}88,3 \\
85,3 \\
79,2 \\
83,5\end{array}$ & $\begin{array}{l}87,2 \\
81,4 \\
77,5 \\
82,8\end{array}$ & $\begin{array}{l}849 \\
85,6 \\
78.5 \\
83,5\end{array}$ & $\begin{array}{l}29 \\
48 \\
1.6 \\
41\end{array}$ & $\begin{array}{c}* \\
\text { N.S. } \\
* \\
\text { N.S. }\end{array}$ \\
\hline $\begin{array}{l}\text { Propionate } \\
\text { Propionate }\end{array}$ & $\begin{array}{l}\text { Estomac } \ldots \\
\text { Iléon } \quad . . \\
\text { Cxcum } \\
\text { Rectum }\end{array}$ & $\begin{array}{l}1,3 \\
0,5 \\
4,4 \\
4,5\end{array}$ & $\begin{array}{l}1,4 \\
0,0 \\
4,4 \\
4,8\end{array}$ & $\begin{array}{l}2.1 \\
0.2 \\
50 \\
5.2\end{array}$ & $\begin{array}{l}49,7 \\
393 \\
22.1 \\
28,7\end{array}$ & $\begin{array}{l}\text { N.S. } \\
\text { N.S. } \\
\text { N.S. } \\
\text { N.S. }\end{array}$ \\
\hline $\begin{array}{l}\text { Butyrate } \\
\text { Butyrate }\end{array}$ & $\begin{array}{l}\text { Estomac } \ldots \\
\text { Iléon } \ldots \\
\text { Cæcum } \ldots \\
\text { Rectum } \ldots\end{array}$ & $\begin{array}{r}52 \\
5.8 \\
13.8 \\
9.0\end{array}$ & $\begin{array}{r}5.5 \\
8,5 \\
16.1 \\
9.5\end{array}$ & $\begin{array}{r}7.2 \\
6.0 \\
14.8 \\
8,4\end{array}$ & $\begin{array}{l}37,9 \\
35,3 \\
15,9 \\
28,0\end{array}$ & $\begin{array}{l}\text { N.S. } \\
\text { N.S. } \\
\text { N.S. }\end{array}$ \\
\hline $\begin{array}{l}\text { Valérate } \\
\text { Valerate }\end{array}$ & $\begin{array}{l}\text { Estomac } \ldots \\
\text { Iléon } \ldots . \\
\text { Cæcum } \ldots \\
\text { Rectum } \ldots\end{array}$ & $\begin{array}{l}3,8 \\
6,0 \\
0,9 \\
1,5\end{array}$ & $\begin{array}{l}5,0 \\
87 \\
0,8 \\
1,8\end{array}$ & $\begin{array}{l}3,9 \\
5,5 \\
1,0 \\
1,9\end{array}$ & $\begin{array}{r}22,8 \\
40,3 \\
2,6 \\
20,5\end{array}$ & $\begin{array}{c}* \\
* \\
* \\
\text { N.S. }\end{array}$ \\
\hline $\begin{array}{l}\text { (1) Rappoit } \\
\text { Ratio be } \\
\text { (2) Effect of } \\
\text { (3) Estomac }\end{array}$ & $\begin{array}{l}\text { l'écart type ré } \\
\text { wilhin-group } \\
\text { diet. } \\
\text { mach; Iléon - }\end{array}$ & $\begin{array}{l}\text { uel intr } \\
\text { idual } \\
\text { eum ; }\end{array}$ & $\begin{array}{l}\text { la } \\
\text { err }\end{array}$ & Rectun & Rectum. & \\
\hline
\end{tabular}


baisse significative de la proportion d'acétate, quand le taux de lignines augmente. La teneur en azote ammoniacal varie également peu en fonction du régime, quel que soit le lieu de prélèvement considéré (tabl. 2). Compte tenu de notre méthode de dosage semi-quantitative, nous ne pouvons pas estimer l'effet moyen du régime sur les teneurs en laciate des digesta. L'absence d'interaction entre les effets "heure de prélèvement» et les effets «aliments» nous permet de grouper les résultats obtenus avec les 3 aliments.

Ainsi, pour les 3 lots et quel que soit l'organe, l'acétate demeure l'AGV le plus représenté suivi du butyrate et du propionate (tabl. 3). Plusieurs travaux ont déjà mentionné la prépondérance du butyrate sur le propionate, que ce soit dans le plasma (Beauville et al., 1974) ou dans le contenu cacal (Hoover \& Heitmann, 1972). Mais le valérate lorsqu'il a été dosé (Susmel \& LANari, 1976) a été trouvé dans de faibles proportions. Nos dosages aux niveaux stomacal et iléal indiquent par contre, que le valérate peut s'y trouver en proportions non négligeables (4 à 7 p. 100), dépassant parfois celles du propionate, voire du buiyrate. De même, dans ces 2 sites, Jes proportions moyennes d'acétate sont élevées, ceci aux dépens du propionate et du butyrate. Dans le cæcum et le rectum, le butyrate et le propionate reviennent à des proportions plus importantes essentiellement aux dépens du valérate, et aussi de l'acétate.

Mais signalons à ce sujet l'importance de l'alimentation sur cette répartition des AGV. Les études antérieures (dont les rations contiennent généralement moins de 6 p. 100 de lignines) présentent des résultats, parfois très variables pour un même site de prélèvement : par exemple, Leng \& Hornicke (1975) trouvent 9 p. 100 de propionate et 86 p. 100 d'acétate dans le contenu iléal, alors que les valeurs obtenues par Wolter et al. (1980) sont respectivement de 80 p. 100 et 15 p. 100.

\section{B. Effets de l'heure du prélèvement}

\section{Acides gras volatils}

Bien que faible $(12,0 \mathrm{mM} / \mathrm{l})$ la teneur en $\mathrm{AGV}$ totaux varie significativement au cours de la journée dans l'estomac (fig. 1), mais elle ne varie pas de manière significative au niveau iléal $(7,4 \mathrm{mM} / 1)$. Très élevée dans le cæcum $(100 \mathrm{mM} / 1)$, cette teneur baisse de 25 p. 100 entre $9 \mathrm{~h}$ et $15 \mathrm{~h}$, puis elle progresse et atteint son maximum entre $21 \mathrm{~h}$ et $24 \mathrm{~h}$. Une telle amplitude de variation a déjà été observée sur des lapins adultes que ce soit par abattage (LE BARS et al., 1971), ou par fistulation iléale et cæcale (LENG \& HoRNICKE, 1975). Dans le rectum, la concentration en AGV totaux augmente de 35 p. 100 entre $6 \mathrm{~h}$ et $9 \mathrm{~h}$, en période d'ingestion de cacotrophes (fig. 1).

En accord avec de nombreux travaux, les variations de l'acidité volatile du cæcum s'accompagnent de variations inverses $(r=-0,78)$ du $\mathrm{pH}$ (tabl. 4). De même, au niveau du fundus, le $\mathrm{pH}$ varie significativement au cours de la journée en relation avec la pratique de la cæcotrophie : la présence des cæcotrophes riches en AGV dans le fundus (entre $12 \mathrm{~h}$ et $15 \mathrm{~h}$ ) abaisse le $\mathrm{pH}$ de moitié.

Nous n'observons aucune différence significative entre les teneurs observées dans l'estomac et l'iléon quel que soit l'AGV (fig. 2). Entre le cæcum et le rectum, la teneur en AGV totaux chute de 50 p. 100 ; la baisse est particulièrement accentuée pour 
le taux de butyrate, qui diminue de 69 p. 100. Cette forte baisse de la teneur en butyrate entre le cæcum et le rectum est en accord avec les résultats de plusieurs auteurs (VERnay \& Raynaud, 1975 ; Bonnafous \& RaYNaud, 1978 ; Wolter et al., 1980).

Sur $24 \mathrm{~h}$, les profils des concentrations des AGV (fig. 2) sont relativement parallèles pour l'acétate et le butyrate dans l'estomac, le cæcum et le rectum, l'iléon montrant peu de variations. Mais ces profils sont différents selon le lieu de prélèvements. Ils reflètent de manière plus accentuée l'évolution des AGV totaux : pics d'acétate et de butyrate lors de l'excrétion des cæcotrophes aux niveaux rectal et stomacal. Les autres AGV varient peu en fonction de l'heure, le valérate par exemple reste à une teneur quasi-constante quels que soient le lieu ou l'heure de prélèvement.
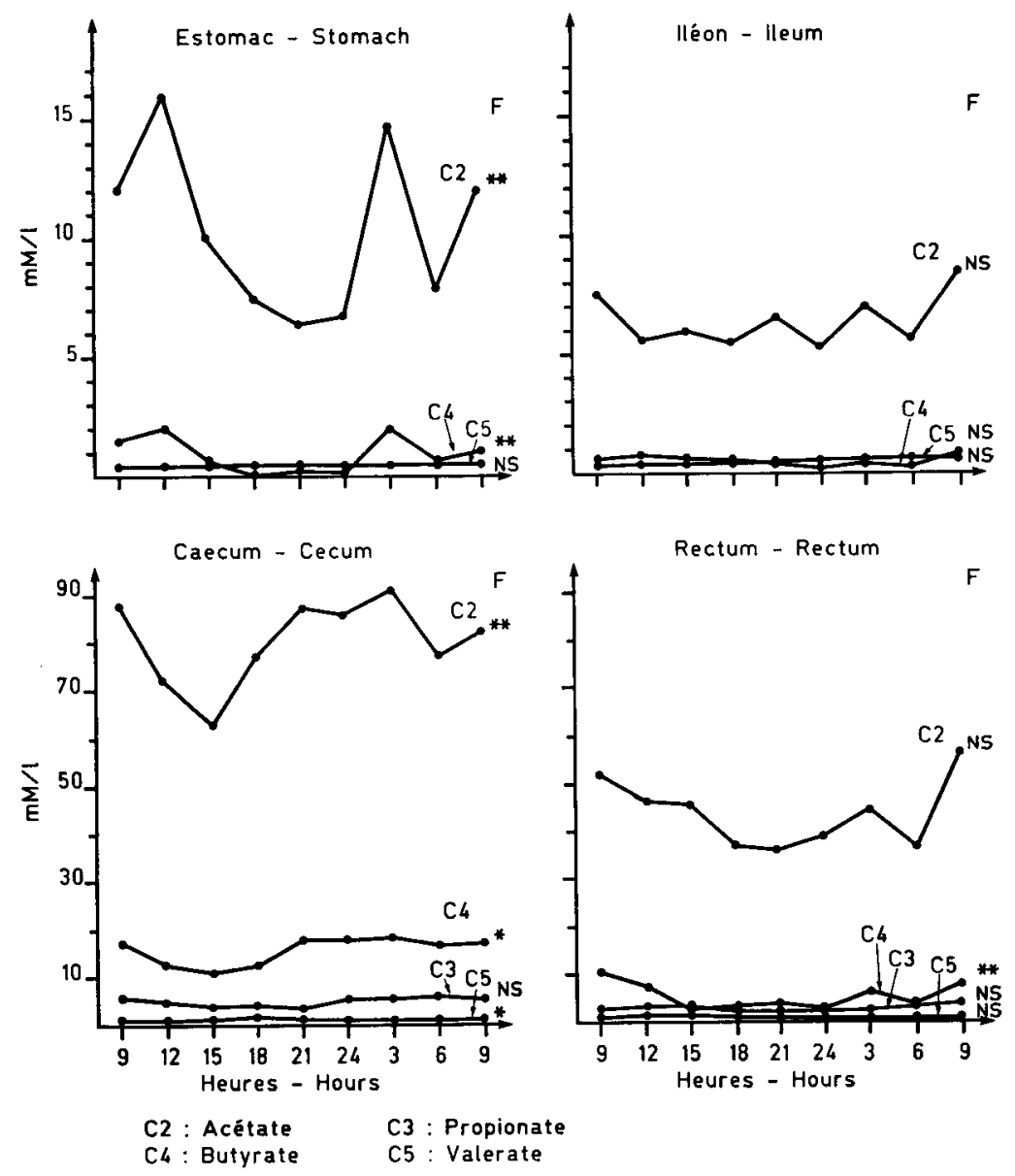

FIG. 2

Evolution nycthémérale de la teneur des principaux $A G V$ en fonction du site de prélèvement.

Circadian variation of main VFA concentration at different collection sites. 


\section{TABleau 4}

Valeurs moyennes du $\mathrm{pH}$ en fonction de l'heure et du lieu de prélèvement. Mean $\mathrm{pH}$ values related to time and collection site.

\begin{tabular}{c|c|c|c}
\hline \multirow{2}{*}{$\begin{array}{c}\text { Heures } \\
\text { Time }\end{array}$} & \multicolumn{2}{|c|}{ Estomac } & Caecum \\
\cline { 2 - 3 } 9 & Fundus & Antrum & \\
\hline 12 & 3,33 & 2,04 & 5,59 \\
15 & 2,34 & 1,75 & 6,00 \\
18 & 1,16 & 1,89 & 5,86 \\
21 & 1,47 & 1,95 & 5,55 \\
24 & 2,06 & 2,20 & 5,54 \\
3 & 1,68 & 2,44 & 5,54 \\
6 & 2,16 & 2,14 & 5,55 \\
9 & 2,61 & 2,12 & 5,63 \\
& 2,34 & 2,14 & 5,73 \\
\hline Moyenne ...... & $2,12 \pm 0,64$ & $2,07 \pm 0,20$ & $5,65 \pm 0,18$ \\
\hline Signif. stat. (F) (1) & $* *$ & N.S. & $* *$ \\
\hline
\end{tabular}

(1) Effect of collection time.

Au cours du nycthémère, les proportions molaires de chaque AGV varient également, mais avec des amplitudes différentes selon l'organe (fig. 3). Ces profils «qualitatifs» restent stables dans l'iléon et le cæcum. L'excrétion des cæcotrophes provoque par contre des variations aux niveaux rectal et stomacal. En effet, les cæcotrophes (récoltés dans le rectum entre $6 \mathrm{~h}$ et $12 \mathrm{~h}$ ) semblent plus riches en butyrate (valeur moyenne 13 p. 100) que les crottes dures (6 p. 100), mais légèrement plus pauvres en acétate (79 p. 100 contre 85 p. 100). VERnaY \& RaYnaud (1975) remarquent aussi, chez le lapin adulte, cette différence de composition en AGV au niveau du côlon proximal et distal selon le type de fèces excrété.

\section{L'azote ammoniacal}

C'est dans l'iléon (tabl. 5) que les concentrations d'azote ammoniacal $\left(\mathrm{N}-\mathrm{NH}_{3}\right)$ sont les plus élevées (valeur moyenne sur $24 \mathrm{~h}: 38,8 \mathrm{mM} / 1$ ) ; elles sont minimum dans l'estomac $(11,8 \mathrm{mM} / 1)$ et ne diffèrent pas significativement pour le cæcum et le rectum $(24,7$ et $22,3 \mathrm{mM} / 1$ respectivement). Ces teneurs sont 2 à 3 fois plus importantes que celles rencontrées dans le cæcum du rat (Demigne et al., 1980) ou le gros intestin du poney (TISSERAND et al., 1977 ; Wolter et al., 1980).

Compe tenu des variations interindividuelles, nous n'observons pas un effet significatif de l'heure de prélèvement, sauf au niveau rectal où, en phase d'excrétion de crottes dures, les digesta sont plus pauvres en $\mathrm{N}^{-\mathrm{NH}_{33}}$ (moyenne de $15 \mathrm{~h}$ à $6 \mathrm{~h}$ : $18,5 \mathrm{mM} / 1$ ) que pendant la phase d'excrétion de cacotrophes (moyenne de $9 \mathrm{~h}$ à $12 \mathrm{~h}: 29,7 \mathrm{mM} / \mathrm{l}$ ). La réduction observée entre le cæcum et le rectum (lors de l'excrétion de crottes dures) indique un phénomène d'absorption, mais moins intense que celui observé dans les travaux de Hoover \& Heitmann (1975). 

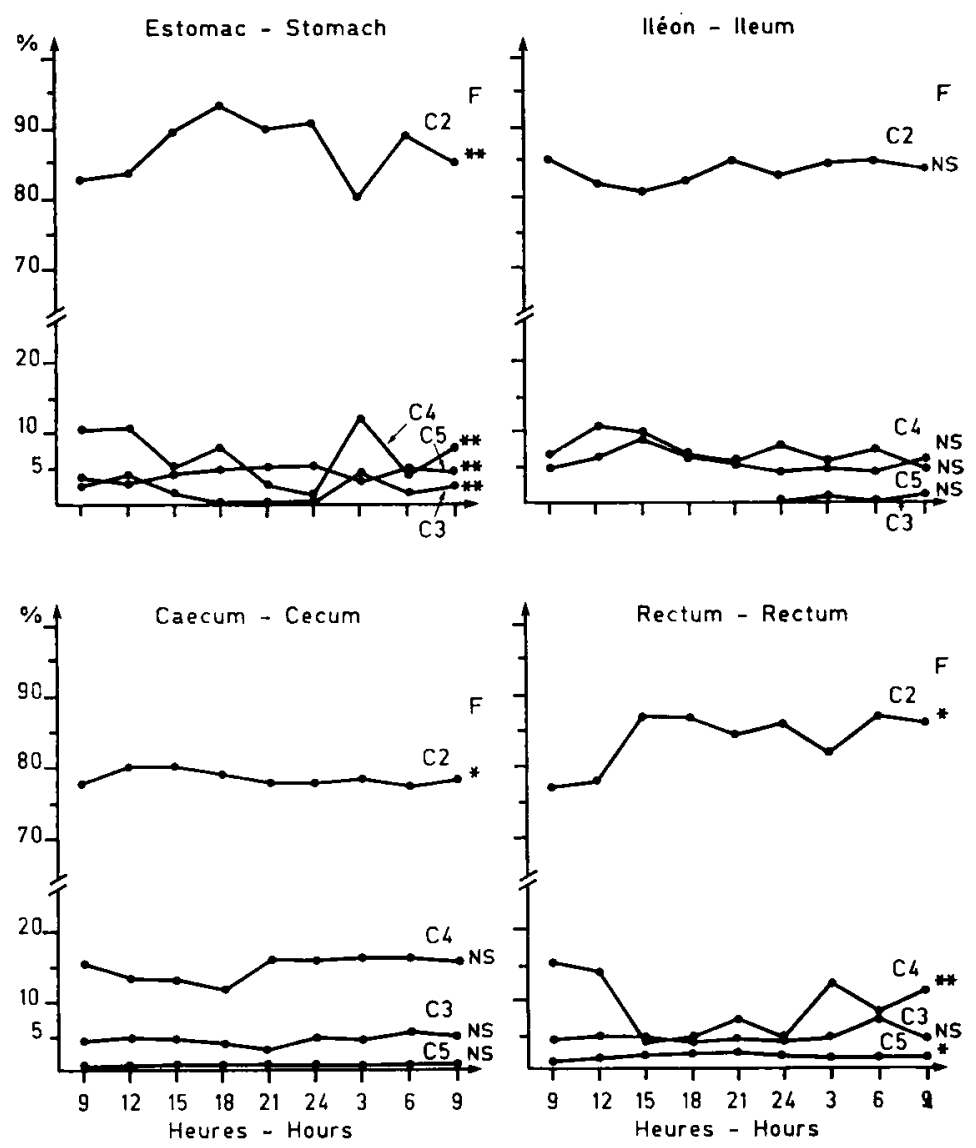

FIG. 3

Evolution nycthémérale des proportions molaires des principaux AGV en fonction du site de prélèvement.

Circadian variation of main VFA molar proportions at different collection sites.

\section{Le lactate}

Etant donné la précision de notre méthode, nous pouvons seulement définir des intervalles de concentration (tabl. 6). Quel que soit le site digestif, la teneur en lactate reste faible $(<10 \mathrm{mM} / 1)$. Le contenu iléal semble être plus riche en lactate que les autres digesta; c'est également dans l'iléon que Wolter et al. (1980) et Vernay (1985) observent les teneurs les plus élevées $(2,4$ à $9 \mathrm{mM} / \mathrm{l})$.

Notre méthode ne nous permet pas de mettre en évidence un effet de l'heure de prélèvement. Signalons toutefois que Vernay (1985) mesure dans les cacotrophes une teneur en lactate $(4 \mathrm{mM} / \mathrm{l})$ très supérieure à celle des crottes dures $(0,4 \mathrm{mM} / \mathrm{l})$, chez des lapins adultes alimentés à base de luzerne et d'avoine. 
Tableau 5

Teneur en ammoniaque ( $\mathrm{mM} / \mathrm{l})$ en fonction de l'heure et du site de prélèvement. Ammonia concentrations $(\mathrm{mM} / \mathrm{l})$ related to time and collection site.

\begin{tabular}{|c|c|c|c|c|c|}
\hline \multirow{2}{*}{$\begin{array}{c}\text { Heures } \\
\text { Time }\end{array}$} & \multicolumn{5}{|c|}{ Organe (site) } \\
\hline & Estomac & Iléon & Cæcum & Rectum & $\begin{array}{c}\text { Effet } \\
\text { organe }\end{array}$ \\
\hline 9 & 12,5 & 38,9 & 32,6 & 33,2 & $* *$ \\
\hline 12 & 11,4 & 40,2 & 23,4 & 33,6 & * \\
\hline 15 & 12,9 & 39,3 & 28,5 & 19,6 & $* *$ \\
\hline 18 & 10,9 & 38,9 & 24,0 & 20,5 & $* *$ \\
\hline 21 & 11,0 & 38,2 & 23,1 & 21,6 & $* *$ \\
\hline 24 & 14,0 & 35,9 & 20,5 & 122 & $*$ \\
\hline 3 & 10,6 & 40,3 & 21,9 & 16,9 & $* *$ \\
\hline 6 & 9,8 & 37,9 & 21,3 & 20,3 & $* *$ \\
\hline 9 & 11,9 & 39,4 & 27,1 & 22,4 & $* *$ \\
\hline Moyenne & $11,7 \pm 1,3^{\mathrm{a}}$ & $38,8 \pm 1,4 b$ & $24,8 \pm 3,3 \mathrm{c}$ & $22,3 \pm 4,0^{\mathrm{c}}$ & $* *$ \\
\hline Effet heure & N.S. & N.S. & N.S. & $*$ & \\
\hline
\end{tabular}

* Les valeurs affectées de lettres différentes sont significativement différentes au seuil $\mathbf{P}<0,01$. Values followed by unlike superscripts are significantly different $(P<0.01)$.

\section{TABLEAU 6}

Estimation semi quantitative du lactate en fonction de l'heure et du site de prélèvement.

Semi quantitative estimation of lactate $(\mathrm{mM} / \mathrm{l})$ related to time and collection site.

\begin{tabular}{c|c|c|c|c}
\hline \hline \multirow{2}{*}{$\begin{array}{c}\text { Heures } \\
\text { Time }\end{array}$} & \multicolumn{3}{|c}{ Organe (site) } \\
\cline { 2 - 5 } 9 & Estomac & Iléon & Cæcum & Rectum \\
\hline 12 & - & $*$ & - & - \\
15 & - & $* *$ & - & - \\
18 & $*$ & $* *$ & - & - \\
21 & - & $*$ & - & $*$ \\
24 & - & $* *$ & - & $*$ \\
3 & $*$ & $*$ & - & - \\
6 & $*$ & $*$ & - & - \\
9 & $*$ & $*$ & - & \\
\hline
\end{tabular}

- : $<1 \mathrm{mM} / 1$.

*: $1-5 \mathrm{mM} / \mathrm{l}$.

** : 5-10 mM/l. 


\section{Discussion générale}

Plusieurs auteurs ont déjà étudié chez le lapin l'effet de la composition du régime, sur le développement des fermentations, et plus particulièrement l'effet de la teneur en glucides pariétaux (Hoover \& HeitmanN, 1972 ; Susmel \& Lanari, 1976). Mais peu de travaux ont étudié les effets de la composition des constituants pariétaux du régime sur les fermentations cæcales.

Nos résultats montrent que les variations de la teneur en lignines influencent peu les produits de la fermentation bactérienne tant sur leur quantité (concentrations) que sur leur qualité (proportions molaires), et quel que soit l'organe considéré. Bien qu'élevées, les teneurs en lignines de nos régimes ne semblent donc pas avoir notablement inhibé la production d'AGV. Nous remarquons même au niveau cæcal une légère hausse de la teneur en $A G V$ totaux, et surtout de l'acétate $(75,0$ - 83,5 $93,1 \mathrm{mM} / 1$ respectivement pour les lots 1,2 et 3 ), lorsque le taux des lignines de la ration augmente. AKIN (1982) signale pourtant que les lignines inhibent la fermentation des «fibres» par la flore du rumen cn raison de l'effet toxique de certains monomères phénoliques composant les lignines (Acide p. coumarique), ou des liaisons lignines-hémicelluloses : JUNG \& FAHEY (1983 b) montrent, in vitro, que l'effet inhibiteur sur les fermentations est variable selon le monomère phénolique. Ces auteurs (JUNG \& FAHEY, 1983 c) observent également in vivo, que la digestibilité des constituants pariétaux est plus fortement inhibée par les lignines des graminées que par celles des légumineuses. La composition chimique de nos rations ne permet pas d'expliquer le phénomène observé. Signalons tout de même que les lignines de nos régimes ont une origine différente de celles utilisées dans l'alimentation des ruminants. Elles proviennent entièrement de luzerne déshydratée (teneur en lignine $=8,2 \mathrm{p}$. 100) dans la ration 1 ; les lignines des rations 2 et 3 proviennent respectivement à 47 et 73 p. 100 de pellicules de colza (teneur en lignine $=31,4$ p. 100). La digestibilité des hémicelluloses $(45$ p. 100$)$ et celluloses $(36$ p. 100) ne diffère pas significativement entre les 3 régimes (GidenNE, 1986, données non publiées). Les faibles écarts (en production d'AGV ou digestibilité) que nous observons, peuvent s'expliquer par une faible résistance des liaisons lignines-hémicelluloses des pellicules de colza, aux agents digestifs présents dans l'intestin du lapin. Il est également possible que, chez le lapin, l'effet inhibiteur n'augmente plus au-delà d'un certain seuil de concentration des lignines dans la ration; seuil inférieur à la valeur minimum utilisée dans notre expérimentation (7,4 p. 100). Quoiqu'il en soit, une analyse des digesta entrant dans le cæcum et une analyse plus fine des lignines (éléments toxiques) et de leurs liaisons avec les autres constituants de la paroi, est nécessaire.

Nos résultats (fig. 2 : rectum 6 h-12 h) montrent que les cacotrophes sont plus riches en AGV que les crottes dures; Vernay (1975) le signale également. Ainsi, l'acidité totale volatile de l'estomac augmente-t-elle lors de l'ingestion des crottes molles, au cours de la matinée. Dans le cæcum, le $\mathrm{pH}$ est minimum et la teneur en AGV maximum entre $24 \mathrm{~h}$ et $3 \mathrm{~h}$ (début de la formation des cacotrophes); LENG \& HoRnicke (1975) observent le même phénomène sur des animaux fistulés.

La «vidange» partielle du cæcum qui précède l'émission des cæcotrophes entraîne une baisse de l'acidité volatile totale dans cet organe (entre $9 \mathrm{~h}$ et $15 \mathrm{~h}$ ). L'activité fermentaire reprend ensuite lorsque le cæcum se remplit avec de nouveaux nutriments. Cette relation entre cæcotrophie et AGV du contenu cæcal est également signalée par Ruckebusch \& Fioramonti (1976) chez des lapins alimentés 2 fois/jour. 
Par ailleurs, à $3 \mathrm{~h}$, certains animaux pratiquent la cæcotrophie. Ceci modifie la teneur en AGV à la fois au niveau de l'estomac, du cæcum et du rectum. Parker (1976) n'observe pas, sur des lapins adultes porteurs de fistule cæcale de telles variations au cours de la journée, et il conclut à l'absence de relation entre la pratique de la cæcotrophie et la production d'AGV. Nos résultats montrent, par contre, une bonne concordance cæcotrophie-AGV que ce soit aux niveaux cæcal, rectal ou stomacal. LE BARs et al. (1971) au niveau du cæcum, Vernay \& RAYNAud (1975) au niveau du côlon, remarquent aussi le même type de variations $(+100$ p. 100 d'acidité volatile pendant l'émission de cæcotrophes).

La dualité de l'excrétion fécale intervient aussi au niveau de l'évolution des proportions molaires des AGV. En période d'excrétion de crottes molles, les proportions des AGV du contenu rectal, sont semblables à celles du cæcum : 75 p. 100 d'acétate, 12 à 15 p. 100 de butyrate, 5 p. 100 de propionate. Le transit du contenu colique est ralenti de 1,5 à 2,5 fois lors de l'excrétion de crottes dures (FIORAmonti \& RUCKEBUSCH, 1976). Ceci autorise des modifications plus profondes des digestas : baisse de la densité bactérienne, plus grande absorption des AGV (CATALA, 1978), et en particulier chute de la proportion de butyrate de 12 à 6 p. 100. Les travaux de Vernay \& MarTy (1984), et de MarTy \& Vernay (1984), soulignent le rôle particulier du butyrate : il diffère des autres AGV par son taux de production, son absorption (passage actif) mais aussi par son apport d'énergie pour le colonocyte. En effet, à la différence du ruminant, la voie métabolique majeure du butyrate n'est pas la production de corps cétonique; mais il peut être entièrement métabolisé (cycle de Krebs) par la paroi colique (surtout en phase d'excrétion de crottes dures). Remesy \& Demigne (1976) montrent aussi chez le rat, une absorption particulière du butyrate au niveau du côlon; ils ajoutent que la cétogenèse au niveau de la paroi cæcale ne concerne que 12 p. 100 du butyrate absorbé et qu'elle semble nulle dans le côlon. Plus globalement, VerNay (1985) montre que les produits de la fermentation microbienne sont aussi bien utilisés chez le lapin (10 à 40 p. 100 de l'énergie d'entretien) que chez le ruminant.

Plusieurs auteurs observent en fonction du régime alimentaire, des variations de la teneur en azote ammoniacal des digestas. Ainsi, Abdel-Rahman \& Shehata (1978), MoRISSE et al. (1985) trouvent des concentrations cæcales plus élevées quand la teneur en fibre de la ration augmente. Mais, les teneurs que nous trouvons dans l'iléon et le cæcum sont nettement plus élevées que celles obtenues par WoLTER et al. en 1980 $(+100$ p. 100), alors que les régimes ont une teneur en cellulose Van-Soest équivalente. A l'inverse Morisse et al. (1985) obtiennent des teneurs en ammoniaque cæcal supérieures aux nôtres $(28,8 \mathrm{mM} / \mathrm{I})$ pour un régime contenant $16 \mathrm{p}$. 100 de cellulose. Au-delà de la teneur globale en fibres du régime, il faut donc chercher d'autres facteurs de variations : par exemple la dégradabilité et la composition (matières azotées, constituants pariétaux) des digestas parvenant au cæcum, qui pourraient entraîner une modification de la flore protéolytique.

Les fortes teneurs en ammoniaque rencontrées dans l'iléon $(38,8 \mathrm{mM} / \mathrm{l})$ ne semblent pas dues à la technique de prélèvement par abattage. En effet, par fistulation iléale (GIDENNE \& Bousssou, 1986) nous obtenons des concentrations en ammoniaque similaires à celles mesurées ici. L'origine de cette accumulation est difficilement attribuable à la flore iléale compte tenu des teneurs en AGV et de la faible densité bactérienne de ce site (Catala, 1978). Aucune donnée bibliographique ne permet d'exclure la possibilité d'un recyclage de l'ammoniaque cæcal au niveau de l'intestin; 
mais CANDAU et al. (1980) suggèrent que l'ammoniac intestinal est essentiellement d'origine gastrique. Aussi, nous pouvons penser qu'un transit rapide des digestas (causé par nos régimes riches en «fibres») diminue l'intensité des phénomènes d'absorption au niveau de l'intestin grêle, et peut expliquer, du moins en partie, les teneurs élevées d'ammoniaque dans l'iléon. De même, un transit accéléré augmente la quantité de matières azotées potentiellement dégradable par la flore cæcale, d'où la richesse en ammoniaque du contenu cæcal de nos animaux $(24,8 \mathrm{mM} / 1$ en moyenne).

Alors que dans l'estomac du rat, le lactate peut atteindre une teneur supérieure à celle de l'acétate (+ de $100 \mathrm{mM} / 1$, selon Remesy \& Demigne, 1976), chez le lapin nos résultats indiquent des teneurs faibles $(<10 \mathrm{mM} / \mathrm{l})$ quelle que soit la zone du tube digestif. Par ailleurs, FonTy (1974) signale chez cet animal l'absence de lactobacilles. Mais bien que faibles, les concentrations en lactate ne sont pas négligeables. Les travaux de Stangassinger et al. (1982) et de Parker \& Mould (1977) indiquent un métabolisme rapide du lactate par la flore cæcale ou gastrique ; la baisse de concentration que nous observons entre l'iléon et le cæcum semble le confirmer. De plus, Alexander \& Chowdhury (1958), ainsi que Hornicke \& Mackiewicz (1976) pensent que les cæcotrophes ont un rôle particulier dans la production de lactate au niveau gastrique : les amylases salivaires et bactériennes dégraderaient une partie de l'amidon ingéré en glucose ; celui-ci diffuserait dans les cæcotrophes, où il serait alors métabolisé par les bactéries. La production de lactate correspondrait alors à une étape intermédiaire du métabolisme bactérien. Dans notre cas, la faible teneur en amidon ( 2 p. 100 M.S.) de nos régimes ne favorise pas la production de lactate; et comme pour l'ammoniaque, un transit rapide peut être la cause de la légère accumulation de lactate dans l'iléon.

\section{Conclusion}

Les régimes que nous avons testés ne nous permettent pas de mettre en évidence un effet simple des lignines de nos régimes expérimentaux sur les fermentations cæcocoliques. Des études complémentaires sont nécessaires (analyse fine des constituants pariétaux de la ration, etc.), pour préciser la sensibilité du lapin vis-à-vis des teneurs en lignines de la ration. La répartition des $3 \mathrm{AGV}$ principaux est différente chez le lapin $(\mathrm{C} 4>\mathrm{C} 3$ ), de celle des polygastriques, mais aussi d'autres monogastriques (rat, porc). L'évolution nycthémérale des teneurs en AGV montre que l'incidence de la pratique de la cæcotrophie se projette sur la quantité et la qualité des fermentations, que ce soit aux niveaux cæcal, rectal ou stomacal.

Reçu en novembre 1985.

Accepté en mars 1986.

\section{Remerciements}

L'auteur tient à remercier J.P. Jouany (INRA, Centre de Theix) pour les dosages d'AGV, de lactate et d'ammoniaque, dans les contenus digestifs. 


\section{Summary \\ Quantitative variation of bacterial fermentation products in the digestive tract of growing rabbits during the 24-h cycle. \\ Relationships with the dietary lignin content}

Threc groups of 36 young rabbits were fed ad libitum diets containing 7 to 17 p. 100 lignin Van-Soest (table 1). After slaughter of the animals every three hours over a 24 -h period, VFA, ammonia nitrogen and lactate were determined in 4 different sites of the digestive tract : stomach, ileum (terminal), caecum and rectum. The lignin content of the experimental diets did not affect the concentrations (table 2) or the proportions (table 3) of caecocolic fermentation products. Ammonia concentration was high in the ileum $(38.8 \mathrm{~mm} / \mathrm{l})$ and caecum $(24.8 \mathrm{mM} / \mathrm{l})$. It increased in the rectum as affected by caecotrophy (table 7), but did not change during the 24-h cycle in the other collection sites. VFA concentrations were maximum in the rectum and caecum $(50-100 \mathrm{mM} / 1)$. Acetate was the major volatile fatty acid (70-90 p. 100), followed by butyrate $(5-16$ p. 100) and propionate $(0-6$ p. 100). During caecotrophy, the total VFA concentration (fig. 1) decreased in the caecum and increased in the stomach and rectum. Caecotrophes collected in the rectum exhibited a higher butyrate concentration than hard faeces and a lower acetate concentration (fig. 3, stomach, rectum).

Key uords : Volatile fatty acids, ammonia, rabbit, caecotrophy, lignin.

\section{Références bibliographiques}

Abdel Rahman M.M., Shehata O., 1978. Effect of high vs low-fibre rations with and without non-protein sources on growth in Baladi rabbits, and production of VFA and ammonia in the alimentary tract. Indian. J. Anim. Sci., 48, 529-533.

Akin D.E., 1982. Microbial breakdown of feed in the digestive tract. « Nutritional limits to animal production from pastures ». Ed. : J.B. HACKER (C.A.B.), symp., 24-28 août 1981. Sainte Lucie, Australie, 201-222.

Akin D.E., Burdick D., Michaels G.E., 1974. Rumen bacterial interrelationships with plant tissue during degradation revealed by transmission electron microscopy. Appl. microbiol., 57, 1149-1156.

Alexander F., Chowdhury A.K., 1958. Digestion in the rabbits's stomach. Br. J. Nutr., 12, 67-73.

Beauville M., Raynaud P., Vernay M., 1974. Concentrations des acides gras volatils plasmatiques chez le lapin. Ann. Rech. vét., 5, 407-411.

Bonnafous R., Raynaud P., 1978. Etude de la concentration des aGV et corps cétoniques plasmatiques au niveau des veines du gros intestin chez le lapin domestique. $2^{*}$ Journées de la Rech. Cunicole, 4-5 avril 1978, ASFC éditeur, communication $\mathbf{N}^{\circ} 5$.

Candau M., Fioramonti J., Touitou M., 1980. Sites de dégradation de l'urée dans le tube digestif du lapin. 2" Congrès Mondial de Cuniculture, Barcelone, avril 1980, Vol. 1, 81-89.

CatalA J., 1978. Recherches sur la physiologie digestive chez le lapin par tine étude expérimentale de la fonction pancréatique. Thèse d'état, Université Paul Sabatier, Toulouse, France, 284 pages.

Cools A., Jeniaux C., 1961. Fermentation de la cellulose et absorption des AGV au niveau du cæcum du lapin. Arch. Int. Physiol. Biochim., 69, 1-8.

Demigne C., Remesy C., Rayssiguier Y., 1980. Effect of fermentable carbohydrates on volatile fatty acids, ammonia and mineral absorption in the rat caecum. Reprod. Nutr. Develop., 20, 1351-1359. 
Fioramonti J., Ruckebusch Y., 1976. La motricité cæcale chez le lapin : III. Dualité de l'excrétion fécale. Ann. Rech. vét., 7, 281-295.

FonTy G., 1974. Etude qualitative et quantitative de la microflore digestive du lapin holoxénique. Thèse $3^{*}$ cycle, Université de Clermont-Ferrand, France, 91 pages.

GidenNE T., LEBAS F., 1984. Evolution circadienne du contenu digestif chez le lapin en croissance. Relation avec la cacotrophie. $3^{\text {e }}$ Congrès Mondial de Cuniculture, Rome, vol. $2,494-501$.

Gidenne T., Bouissou T., 1986. Création d'une fistule iléale chez le lapin adulte. Caractérisation des digesta prélevés. Reprod. Nutr. Dévelop., publication en cours.

HoOver W.H., HeITMANN R.N., 1972. Effects of dietary fiber on weight gain, cecal volume and volatile fatty acid production in rabbits. J. Nutr., 102, 375-379.

HoOver W.H., HeItManN R.N., 1975. Cecal nitrogen metabolism and amino acid in the rabbit. J. Nutr., 105, 245-252.

Hornicke H., Mackiewicz A., 1976. Production d'amylase, décomposition de l'amidon et formation des acides $D$ et $L$ lactiques par les cacotrophes. $1^{\text {er }}$ Congrès Int. cunicole, Dijon, France. Communication n" 56.

JOUANY J.P., 1982. Dosages des acides gras volatils (AGV) et des alcools dans les ensilages par chromatographie en phase gazeuse. Bull. Tech. CRVZ Theix, INRA, 46, 63-66.

JUNG H.G., FAHEY G.C., 1983 a. Nutritional implications of phenolic monomers and lignin : a review. J. Anim. Sci., 57, 206-219.

Jung H.G., FAHEY G.C., 1983 b. Inhibitory effects of phenolic monomers on fermentation of structural carbohydrates by rumen microorganisms. J. Anim. Sci., 57, supplément n" 1, 287-288, abstract $\mathrm{n}^{\circ} 381$.

Jung H.G., FAHEY G.C., 1983 c. Effect of ruminant digestion and metabolism on phenolic monomers of forages. Br. J. Nutr., 50, 637-651.

Le Bars H., Guemon L., Demaux G., 1971. Production d'acides gras volatils dans le cæcum du lapin. Ann. Biol. anim. Biochim. Biophys., 11, 301-302 (Abstr.).

LENG E., HoRNICKE H., 1975. Tagesrhythmische Unterschiede in der Zusammensetzung des Blinddarminhalts von Kaninchen. $Z$. Versuchstier $K$., Bd, 17, 285-299.

MarTy J., Vernay M., 1984. Absorption and metabolism of the volatile fatty acids in the hind-gut of the rabbit. Br. J. Nutr., 51, 265-277.

Morisse J.P., Bollletot E., Maurice R., 1985. Alimentation et modification du milieu intestinal chez le lapin (AGV, $\mathrm{NH}_{3}$, pH, flore). Rec. Med. Vet., 161, 443-449.

PARKER D.S., 1976. Turnover of volatile fatty acids in the caecum of the rabbit. $1^{\text {er }}$ Congrès intern. cunicole, Dijon, France. Communication $\mathrm{n}^{\circ} 51$.

Parker D.S., Mould A.J., 1977. The metabolism of lactic acid in the large intestine of the rabbit. Proc. Nutr. Soc., 35, 4A.

Remesy C., Demigne C., 1976. Partition and absorption of volatile fatty acids in the alimentary canal of the rat. Ann. Rech. vét., 7, 39-55.

Ruckebusch Y., Fioramonti J., 1976. Sur le déterminisme de la cacotrophie chez le lapin. $1^{\text {er }}$ Congrès Int. Cunicole, Dijon, France, Communication $\mathrm{n}^{\circ} 54$.

Stangassinger M., Wallenberg P.V., Giesecke D., 1982. Caecotrophy dependent changes in the metabolic fate of D (-) lactic-acid in rabbits. Arch. Intern. Physiol. Biochim., 90, 347-354.

Susmel P., LANaRi D., 1976. Changes in volatile fatty acid level in rabbit caecum. $1^{\text {er }}$ Congrès intern. cunicole, Dijon, France. Communication $\mathrm{n}^{\circ} 32$.

Tisserand J.L., Masson C., Ottin-Pecchio M., Creusot A., 1977. Mesure du pH et de la concentration en AGV dans le cæcum et le côlon du poney. Ann. Biol. anim. Biochim. Biophys., 17, 553-557.

VAn Soest P.J., Wine R.H., 1967. Use of detergents in the analysis of fibrous feed. IV. Determination of plant-wall constituents. J. Ass. Off. Agric. Chem., 50, 50-55.

VERNAY M., 1985. L'absorption et le devenir des acides gras volatils digestifs chez le lapin, en relation avec la dualité de l'émission fécale. Thèse d'état, Université Paul Sabatier, Toulouse, France. 266 pages.

Vernay M., Raynaud P., 1975. Répartition des acides gras volatils dans le tube digestif du lapin domestique. I. Lapins alimentés en luzerne et avoine. Ann. Rech. vét., 6, 357-368.

Vernay M., Marty J., 1984. Absorption and metabolism of butyric acid in rabbit hind-gut. Comp. Biochem. Physiol., 77 A, 89-96.

Weatherburn M.W., 1967. Phenol-hypochlorite reaction for determination of ammonia. Analyt. chem., 39, 971-974.

Wolter R., Nouwakpo F., Durix A., 1980. Etude comparative de la digestion d'un aliment complet chez le poney et le lapin. Reprod. Nutr. Dévelop., 20, 1723-1730. 